\title{
Transposition and Voltage Unbalance in High Phase Order Power Transmission Systems
}

Brian J. Pierre

Gerald T. Heydt

Arizona State University

Tempe, Arizona USA

\begin{abstract}
High phase order (HPO) systems have been proposed at the early inception of power transmission engineering, but few direct applications have been made. High phase order transmission should be considered as an alternative in the case of high power density applications. In this paper an analysis of transposition of HPO overhead transmission lines is presented and voltage unbalance in HPO systems is considered. Definitions are presented for 'fully transposed' and 'roll transposed' along with advantages and disadvantages of each. A generalized voltage unbalance factor (VUF) is introduced and utilized to determine the benefits of transposition. The generalized VUF is compared with three other possible unbalance factors to determine if the generalized VUF is an appropriate indication of unbalance. Exemplary results are presented for six phase and twelve phase designs. Conclusions show that the generalized VUF is a good indication of transmission line voltage unbalance and certain configurations may not need full rotation transposition to minimize the unbalance factor. The transposition analysis and voltage unbalance are considerations in the assessment of HPO as a high power transmission alternative.
\end{abstract}

Keywords Power transmission, transmission engineering, high phase order, six phase, transposition, voltage unbalance factor.

Received January 2014.

The authors acknowledge the support of the Power Systems Engineering Research Center (PSerc) which is a Generation III National Science Foundation Industry University Cooperative Research Center, awards EEC-0001880, EEC-0968993. The authors also acknowledge the support of the U.S. Department of Energy for its PSerc future grid initiative.

The authors are with the School of Electrical, Computer, and Energy Engineering at Arizona State University, Tempe, AZ 85287, and can be reached at $\{$ brian.pierre, heydt $\}$ asu.edu. 


\section{Introduction}

This paper relates to high phase order $\left(n_{\varphi}>3\right)$ power transmission with a focus on transposition and voltage unbalance. Although there are presently no bulk power applications of HPO systems, there have been demonstration studies [1-5] and various other studies [6-11]. It is also possible that high phase order loads (that is loads served directly at high phase order, e.g., induction motors [12-14]) could be served directly from HPO distribution circuits. It is postulated that when considering all alternatives for bulk power transmission, innovative concepts such as high phase order should be included. Limited right of way, economic issues, and environmental issues also come into play in transmission engineering, and some of these factors may favor high phase order. There are many advantages and disadvantages to HPO designs; these are not discussed in this paper, but can be found in the literature, e.g. [15]. This paper will focus on HPO transposition and voltage unbalance.

Transposition of overhead AC transmission circuits has been used for decades. Transposing three phase lines has been studied extensively, e.g., $[16,17]$. However, transposing HPO lines is infrequently discussed. The reasons for transposing lines can be found in the literature, e.g. [18-20]. In brief, untransposed transmission lines cause voltage and current unbalance that lead to problematic operating conditions, counter rotating torque on machines, increased losses in some cases, and protection considerations. For analysis purposes this paper focuses only on unbalance caused by the transmission line (i.e., self impedances and mutual coupling among the phases).

The term 'fully transposed' may take on a range of meanings. For present purposes, assume that a fully transposed transmission line has a line impedance matrix in the form,

$$
Z_{p h}=\left[\begin{array}{cccc}
S & M & \cdots & M \\
M & S & & \vdots \\
\vdots & & \ddots & M \\
M & \cdots & M & S
\end{array}\right]
$$


where, $S$ is the self impedance, and $M$ is $j$ times the mutual reactance between phases. It may be possible to include resistance in the mutual terms, but this complication is omitted. The shunt capacitance is readily modeled through the use of a capacitance matrix: the capacitance matrix is in the same form as (1) with the capacitance to ground on the diagonals and the phase-phase capacitance on the off-diagonals. For the fully transposed case, the off-diagonal entries of the capacitance matrix are all equal and the diagonal entries are all equal. In (1), $Z_{p h}$ is defined in terms of the line terminal voltages $V_{l}$ and $V_{2}$, and the line current $I$. For an $n$ phase circuit,

$$
V_{l}-V_{2}=Z_{p h} I
$$

The $V$ and $I$ vectors are complex $n$-vectors, and the $Z_{p h}$ matrix is a complex, symmetrical $n$ by $n$ matrix.

The term 'roll transposed' refers to a transmission line that is physically 'rolled' as pictorially illustrated for a six phase example in Fig. 1. Fig. 1 may be in any configuration and any number of phases, the same roll transposition method applies to render the capacitance matrix and impedance matrix in the $n$ by $n$ symmetrical circulant Toeplitz (SCT) form,

$$
Z_{p h}=\left[\begin{array}{cccccccc}
S & M_{1} & M_{2} & \ldots & M_{n / 2} & \ldots & M_{2} & M_{1} \\
M_{1} & S & M_{1} & \ddots & & \ddots & & M_{2} \\
M_{2} & M_{1} & S & \ddots & & & & \vdots \\
\vdots & \ddots & \ddots & \ddots & & & & M_{n / 2} \\
M_{n / 2} & & & & & & & \vdots \\
\vdots & \ddots & & & & & & M_{2} \\
M_{2} & & & & & & & M_{1} \\
M_{1} & M_{2} & \ldots & M_{n / 2} & \ldots & M_{2} & M_{1} & S
\end{array}\right]
$$

for even $n$. For odd $n, Z_{p h}$ is in the $n$ by $n$ SCT form, 


$$
Z_{p h}=\left[\begin{array}{ccccccccc}
S & M_{1} & M_{2} & \ldots & M_{(n-1) / 2} & M_{(n-1) / 2} & \ldots & M_{2} & M_{1} \\
M_{1} & S & M_{1} & \ldots & \ldots & M_{(n-1) / 2} & \ddots & \ldots & M_{2} \\
M_{2} & M_{1} & S & \ddots & & & \ddots & & \vdots \\
\vdots & \ddots & \ddots & \ddots & & & & \\
M_{(n-1) / 2} & \ldots & & & & & & M_{(n-1) / 2} \\
M_{(n-1) / 2} & \ddots & & & & & & & M_{(n-1) / 2} \\
\vdots & \ddots & & & & & & & \vdots \\
M_{2} & & & & & & & & M_{2} \\
M_{1} & M_{2} & \ldots & M_{(n-1) / 2} & M_{(n-1) / 2} & \ldots & M_{2} & M_{1} & S
\end{array}\right]
$$

where $M_{i}$ is $j$ times the mutual reactance determined by the distance between the corresponding two conductors, $d_{i}$, as shown in Fig. 2. In Fig. 2, only circularly symmetric configurations are shown, i.e., the conductors are located on a circumscribed circle at points 360/n degrees apart. Circulant matrices have special properties that are discussed in $[21,22]$. For three phase systems, roll transposed conductors are fully transposed; however, for HPO systems this observation is not true.

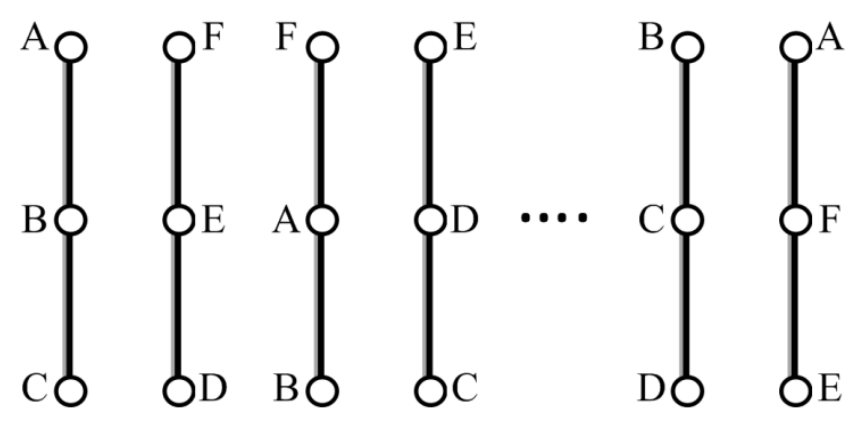

Fig. 1 An illustration of 'rolling' the phases of a six phase transmission line in a transposition process

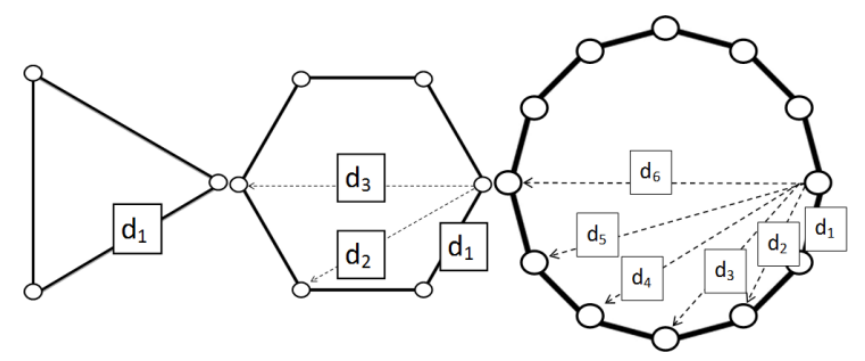

Fig. 2 Circularly symmetric transmission line conductor configuration examples for $n=3,6,12$ 


\section{A comparison of fully transposed vs. roll transposed transmission lines}

The fault analysis for a fully transposed line is straightforward because all the sequence impedances are equal (other than the zero sequence impedance). This property results in relatively simple protection schemes for fewer fault types as compared to the rolled transposition case. However, there are disadvantages to fully transposing an HPO transmission line.

HPO transmission lines have lower phase to phase voltage than comparable three phase counterparts. This lower voltage permits closer phase conductor spacing. The closer phase spacing results in potential benefits [15]. If HPO lines were fully transposed, the phase compaction benefits may be lost: this is due to the fact that greater phase spacing is needed between phases $A$ and $C$ than $A$ and $B$ (for example) in HPO lines. For this reason high phase order transmission lines should be roll transposed, not fully transposed. In addition, roll transposed designs are relatively simple from a construction point of view, whereas fully transposed designs require exchanging conductors and different tower configurations. Perhaps the most salient reason that roll transposition is preferred over full transposition is that the number of transposition sections needed is dramatically reduced in the case of roll transposition. Table I shows the number of transposition sections required for differently configured transmission lines to be either roll transposed or fully transposed.

In Table I the configuration of most interest is the circularly configured case because this design makes the best use of higher power transfer with limited right of way (ROW). Double vertical or vertically configured HPO transmission lines are also of interest because they can transfer high power with shorter tower heights as compared to comparable multicircuit three phase designs. 
TABLE I CONDUCTOR CONFIGURATIONS FOR TRANSPOSITION

SECTIONS FOR FULL TRANSPOSITION ( $n=$ NUMBER OF PHASES)

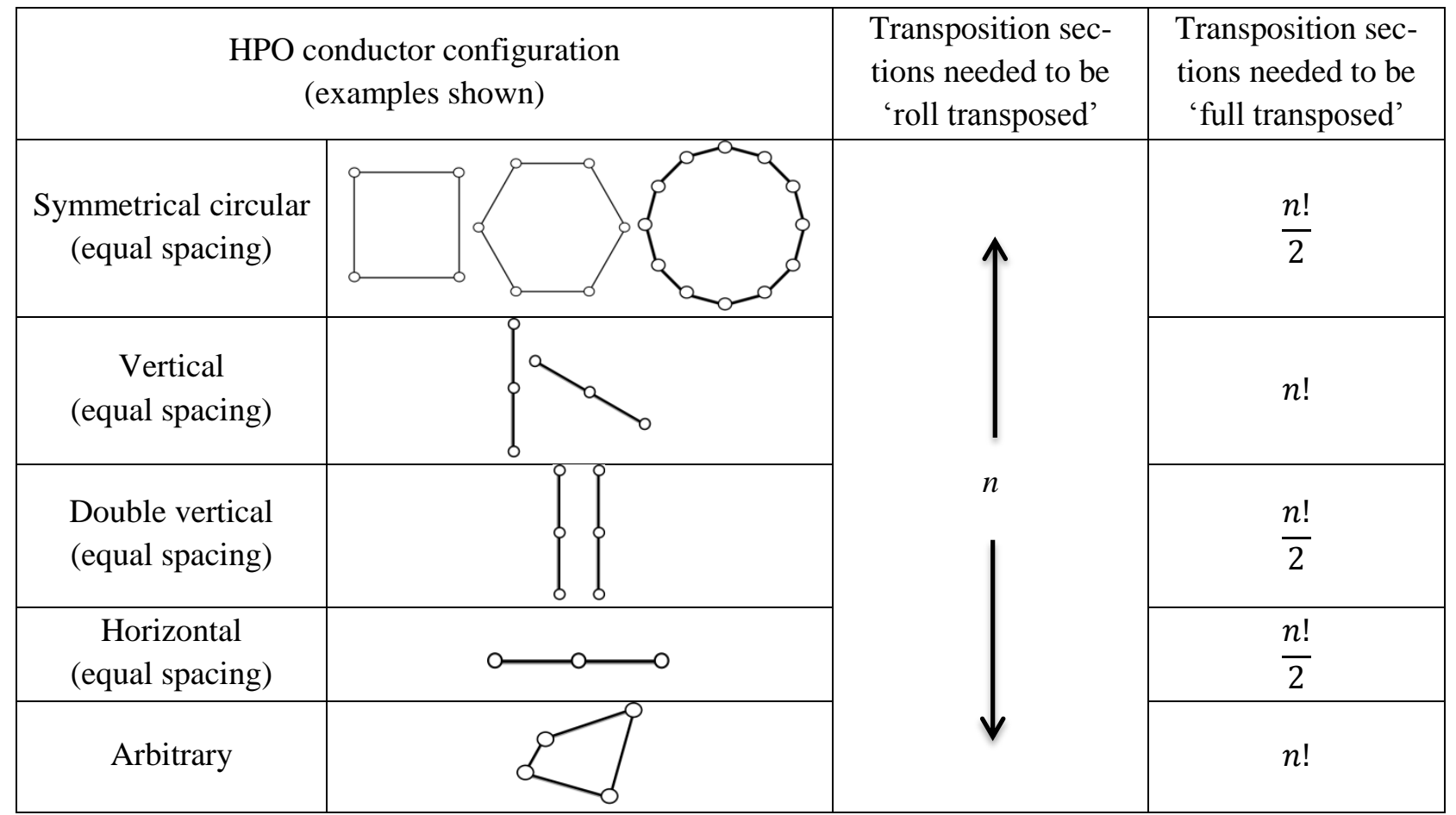

\section{Voltage unbalance factor}

A commonly accepted unbalance factor for three phase voltages is,

$$
U_{3 p h}=\frac{\left|V_{-}\right|}{\left|V_{+}\right|}
$$

with $V_{-}$and $V_{+}$denoting the negative and positive sequence voltages. Equation (4) will be termed the 'three phase voltage unbalance factor' for purposes of this paper. Other common voltage unbalance factors are defined by NEMA and IEEE [23] and further unbalance factors are discussed in [18, 20]. For $n_{\varphi}$ circuits there are $n_{\varphi}$ sequence voltages. Certain other sequence voltages that are similar to negative sequence voltage in the three phase case may result in undesired torque in rotating machines. For this reason, it appears to be useful to define an $n_{\varphi}$ VUF that models the positive sequence voltage in the denominator and all other sequences in the numerator. Therefore, a 'generalized voltage unbalance factor' is introduced, 


$$
U_{n p h}=\frac{\sqrt{\sum_{q=2}^{n-1}\left|V_{q}\right|^{2}+\left|V_{0}\right|^{2}}}{\left|V_{1}\right|}
$$

where $n$ is the number of phases, zero sequence voltage is $V_{0}$, positive sequence voltage is $V_{l}$, negative sequence voltage is $V_{n-1}$ and the other sequences $1<q<n-1$ are sequences that do not exist in the three phase case. To measure unbalance for an HPO transmission line the generalized VUF will be compared with three other possible measures of VUF. In this comparison it will be shown that the generalized VUF is a good indication of unbalance for an HPO transmission line.

The generalized VUF is used in this paper to illustrate the consequences of transposition and geometry of the conductors for HPO transmission lines. A possible use of HPO is to supply HPO motors directly. Therefore, an HPO complex voltage unbalance factor (CVUF) could be useful to determine the derating of HPO motors with voltage unbalance. CVUFs are discussed in the literature [24, 25]. However, it should be noted that even a CVUF may not be a sufficient indication of voltage unbalance [26, 27].

\section{Voltage unbalance factor as a measure of transposition effectiveness}

Transposition is needed in long line designs. However, transposition is expensive and can cause stress on conductors (e.g., mile for mile, faults are more likely in transposition sections than ordinary sections [28], and mechanical stress occurs in transposition sections). For these reasons, it is beneficial to determine the number of transpositions needed to minimize the VUF to an acceptable level. If the transmission line is completely roll transposed, the VUF due to the transmission line impedances is zero.

The four VUFs compared are calculated in a way described in the flow chart in Fig. 3. For phase order $n=3 k$ where $k=1,2, \ldots$, a way to measure voltage unbalance for an HPO transmission line is to utilize the voltage unbalance of each three phase circuit that may be derived from the $k$ different three phase subcircuits. For example, for a six-phase transmission line, measurements of unbalance would be taken 
from two three phase circuits, i.e., unbalance on phases A, C, E, and unbalance on phases B, D, F. Both the maximum unbalance and the average unbalance are analyzed and compared to the generalized VUF.

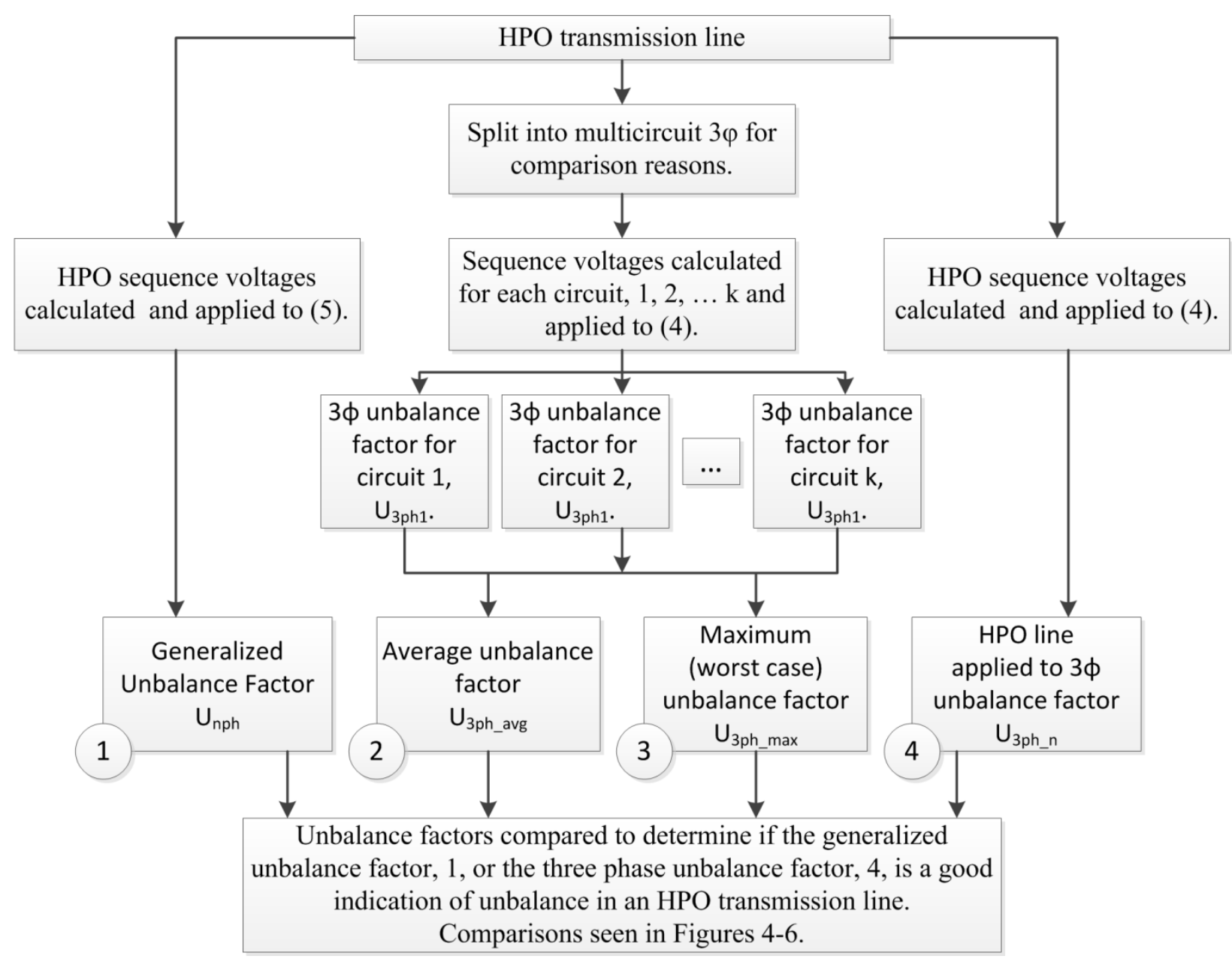

Fig. 3 Flow chart to calculate four unbalance factors for comparison purposes

A VUF comparison will be made for the following test bed example, however note that similar examples follow the same trends and results. A long line example is used to emphasize the impact of transposition on voltage unbalance. Consider a 200 mile long six phase $79.7 \mathrm{kV}$ voltage line-line $\left(V_{l l}\right)$ transmission line and a twelve phase $41.2 \mathrm{kV} V_{l l}$ transmission line; this will be equivalent to a three phase $138 \mathrm{kV} V_{l l}$ transmission line. All example lines will carry $100 \mathrm{MVA}$ of power at 0.8 power factor. Drake conductors will be utilized with $5 \mathrm{ft}$. phase to phase spacing. The height of the lowest conductor is 35 feet. Examples will be shown for six phase and twelve phase designs in configurations shown in Table II [15]. Dashed 
lines are insulators. The VUFs are calculated for each number of transposition sections applied to the example transmission lines, and the VUFs are compared in Figures 4-6.

Table II Different HPO configurations for the cited test bed example

(Drake, 200 mile, 100 MVA line)

\begin{tabular}{|c|c|c|c|c|}
\hline $\begin{array}{c}\text { Configuration A } \\
\text { six phase circular }\end{array}$ & $\begin{array}{c}\text { Configuration B } \\
\text { six phase double } \\
\text { vertical }\end{array}$ & $\begin{array}{c}\text { Configuration C } \\
\text { six phase verti- } \\
\text { cal }\end{array}$ & $\begin{array}{c}\text { Configuration D } \\
\text { twelve phase } \\
\text { circular }\end{array}$ & $\begin{array}{c}\text { Configuration E } \\
\text { twelve phase } \\
\text { double vertical }\end{array}$ \\
\hline & & $\ldots .$. & $\ldots$ & $\ldots$ \\
\hline
\end{tabular}

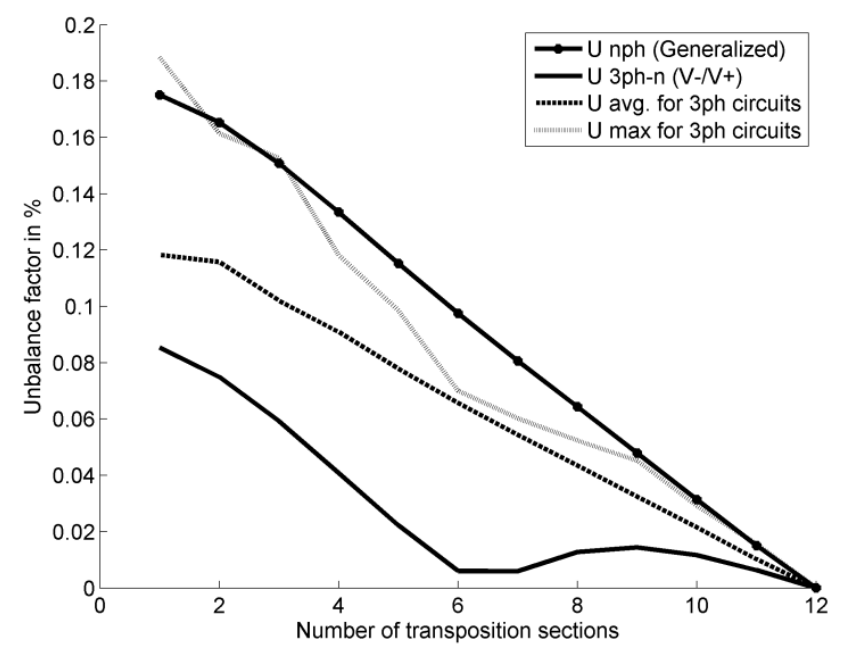

Configuration D: Twelve phase circular

Notice how low the VUF is to begin with. For this reason it is possible that no transposition is needed for circular designs.

Notice the generalized VUF is similar to the avg. of the four individual three phase circuits VUF and the maximum of the four individual three phase circuits VUF.

Notice how the unbalance factor for 3 phase applied to 12 phase is not a good indication of unbalance (solid line).

Configuration A is very similar to this case, and therefore not shown.

Fig. 4 VUF vs. number of transpositions sections for Configuration D (from Table II) 


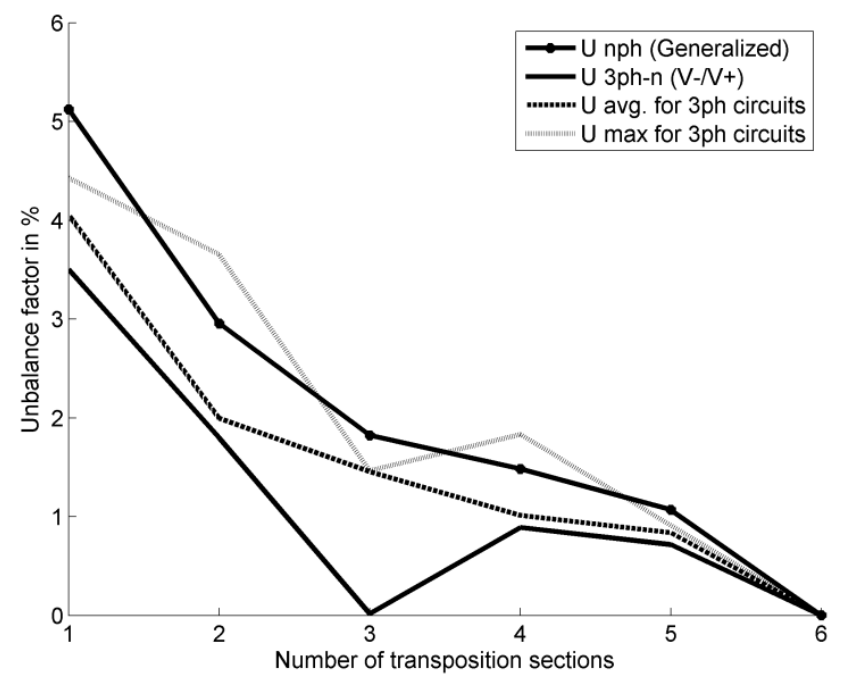

Configuration C: Six phase vertical

Notice the generalized unbalance factor is similar to the average of the two individual three phase circuits unbalance factor and the maximum of the two individual three phase circuits unbalance factor.

Fig. 5 VUF vs. number of transpositions sections for Configuration C (from Table II).

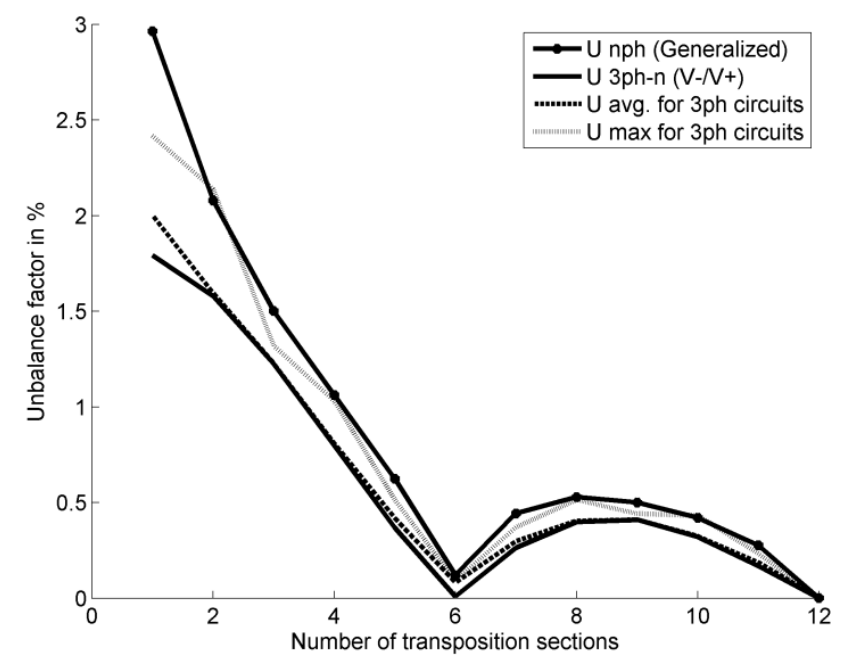

Configuration E: twelve phase double vertical

Notice that after $n / 2$ transposition the unbalance is the second lowest.

Notice how the generalized VUF is similar to the other measurements of VUF. Again showing that the generalized VUF is a good indicator of voltage unbalance.

Configuration B is very similar to this case, and therefore not shown.

Fig. 6 VUF vs. number of transpositions sections for Configuration E (from Table II).

Note that results were also checked with NEMA and IEEE voltage unbalance factors, and similar results were verified. The generalized VUF followed the same trends and similar magnitudes as the NEMA and IEEE VUFs. A few key results can be drawn from Figs. 4-6:

A. From these results it can be shown that generally for the first $n / 2$ transpositions, with increase in transposition sections there is a decrease in VUF. Therefore one can determine if all transpositions are needed, or if it is more economical to use just enough transposition sections to reach an acceptable unbalance. 
B. Notice that if the transmission line is circularly configured the VUF is very small and may not need any transposition.

C. Notice if the transmission line is double vertically configured, after $n / 2$ transpositions, the VUF is often the second lowest. For this reason, it may be beneficial to use $n / 2$ roll transposition sections instead of fully rotating the conductors and using $n$ roll transpositions.

D. Notice that the defined generalized VUF is similar and corresponds to the average of the multicircuit three phase VUF and the maximum of the multicircuit three phase VUF. The generalized VUF is also shown to be a better indicator than using the three phase VUF applied to the HPO case. Therefore, an appropriate indication of voltage unbalance can be obtained with (5) without additional steps of converting to multicircuit three phase.

E. If a $3 k$ order circuit $(k=1,2, \ldots)$ energizes $k$ three-phase rotating loads, the maximum of the multicircuit three phase VUF will give a measure of the greatest impact on the three phase rotating loads $[29,30]$ and rectifier loads [31]. The generalized VUF also affords an appropriate impact measure. Further studies may also research a CVUF to give a better indication of the effects of voltage unbalance on rotating loads $[24,25]$.

\section{Conclusions}

Although there are no HPO transmission lines in existence, it is beneficial to study the possibility of HPO in the design of high density power transmission. This paper discusses two aspects of HPO transmission: transposition and voltage unbalance. Definitions of 'roll transposed' and 'fully transposed' have been presented with advantages and disadvantages of each. The conclusion is that roll transposition should be used with HPO transmission lines that have high unbalance. In addition, a generalized voltage unbalance factor was introduced and verified through comparison to other unbalance measures. The generalized unbalance factor is an appropriate indication of voltage unbalance for an HPO transmission line and a better indication than using the traditional three phase unbalance factor. Fully rotating the phases in roll transposition may not be needed, that is, it may be sufficient to use just enough transposition sections 
to minimize the unbalance factor to an acceptable level. This is especially true for double vertical constructions where $n / 2$ transposition sections may sufficiently attain a low level of voltage unbalance.

\section{References}

[1] M. Brown, R. Rebbapragada, T. Dorazio, J. Stewart, "Utility system demonstration of six phase power transmission," Proc. IEEE Transmission and Distribution Conference, Dallas, TX, 1991.

[2] New York State Electric \& Gas Corporation, "High phase order transmission demonstration: Final report,” Empire State Electric Energy Research Corporation, Albany NY, 1997.

[3] J. Stewart, L. Oppel, G. Thomann, T. Dorazio, M. Brown, "Insulation coordination, environmental, and system analysis of existing double circuit line reconfigured to six-phase operation," IEEE Transactions on Power Delivery, Vol. 7, No. 3, pp. 1628-1633, 1992.

[4] A. Apostolov, R. Raffensperger, "Relay protection operation for faults on NYSEG's six-phase transmission line,” IEEE Transactions on Power Delivery, Vol. 11, No. 1, pp. 191-196, 1996.

[5] R. Rebbapragada, H. Panke, H. Pierce, J. Stewart, L Oppel, "Selection and application of relay protection for six phase demonstration project," IEEE Transactions on Power Delivery, Vol. 7, No. 4, pp. 1900-1911, 1992.

[6] H. C. Barnes, L. O. Barthold, "High phase order power transmission," CIGRE SC 32, Electra, No. 24, 1973.

[7] T. Dorazio, "High phase order transmission," Proc. Southern Tier Technical Conference, Binghamton, NY, 1990.

[8] T. Landers, R. Richeda, E. Krizanskas, J. Stewart, R. Brown. "High phase order economics: constructing a new transmission line," IEEE Transactions on Power Delivery, Vol. 13, No. 4, pp. 1521-1526, 1998.

[9] N. Bhatt, S. Venkata, W. Guyker, W. Booth, "Six-phase (multi-phase) power transmission systems: Fault analysis," IEEE Transactions on Power Apparatus and Systems, Vol. 96, No. 3, pp. 758-767, 1977. 
[10] W. Abu-Elhaija, F. Amoura, "Fault analysis of six phase power system using six phase symmetrical components," Electric Power Components and Systems, Vol. 33, No. 6, pp. 657-671, 2005.

[11] M. Farghaly, E. Ibrahim, "An important study of the transverse faults on twelve-phase power systems," Electric Power Components and Systems, Vol. 34, No. 4, pp. 369-383, 2006.

[12] E. A. Klingshirn, "High phase order induction motors part I- description and theoretical considerations," IEEE Transactions on Power Apparatus and Systems, Vol. 102, No. 1, pp. 47 - 53, 1983.

[13] E. E. Ward, H. Härer, "Preliminary investigation of an inverter fed 5-phase induction motor," Proc. IEE, Vol. 116(B), No. 6, pp. 980 - 984, 1969.

[14] A. Iqbal, G. Singh, V. Pant, "Steady-state modeling and analysis of six-phase synchronous motor," Systems Science \& Control Engineering, Vol. 2, No. 1, pp. 236-249, 2014.

[15] J. Bortnik, 'Transmission line compaction using high phase order transmission,' doctoral thesis, University of the Witwatersrand, Johannesburg, South Africa, 1998.

[16] E. Gross, S. Nelson, "Electromagnetic unbalance of untransposed transmission lines," IEEE Transactions on Power Apparatus and Systems, Vol. 74, No. 3, pp. 887-893, 1955.

[17] J. Ma, S. Fortin, F. Dawalibi, "Analysis and mitigation of current unbalance due to induction in heavily loaded multicircuit power lines," IEEE Transactions on Power Delivery, Vol. 19, No. 3, pp. 1378-1383, 2004.

[18] J. Mooney, "Economic analysis and justification for transmission line transposition," Proc. IEEE Transmission and Distribution Conference and Exposition, New Orleans, 2010.

[19] M. Hesse, "Circulating currents in paralleled untransposed multicircuit lines: I- numerical evaluations," IEEE Transactions on Power Apparatus and Systems, Vol. 85, No. 7, pp. 802-811, 1966.

[20] T. Chen, "Evaluation of line loss under load unbalance using the complex unbalance factor," IEEE Proc. Generation, Transmission and Distribution, Vol. 142, No. 2, pp. 173-178, 1995.

[21] G. Heydt, B. Pierre, "Integrating transmission and distribution engineering eventualities," Proc. PSERC Future Grid Initiative, pp. 63-68, Madison, WI, 2013. 
[22] R. Gray, Toeplitz and circulant matrices: A review, Foundations and Trends in Communications and Information Theory, Now Publishers, Hanover MA, 2006.

[23] J. Kim, E. Lee, D. Lee, J. Lee, "Comparison of voltage unbalance factor by line and phase voltage," International Conference Proc. on Electrical Machines and Systems, Vol. 3, pp. 1998-2001, 2005.

[24] Y. Wang, "Analysis of effects of three-phase voltage unbalance on induction motors with emphasis on the angle of the complex voltage unbalance factor," IEEE Transactions on Energy Conversion, Vol. 16, No. 3, pp. 270-275, 2001.

[25] C. Reineri, J. Gomez, N. Campetelli, “Adjustable speed drives supplied with unbalanced voltages: Study under complex voltage unbalance factor parameter," IEEE Proc. on Transmission and Distribution Conference and Exposition, Bergamo, Italy, 2008.

[26] A. Filho, et al. "Analysis of the complex voltage unbalance factor behavior resulting from the variation of voltage magnitudes and angles," IEEE International Conference Proc. on Harmonics and Quality of Power, Bergamo, Italy, 2010.

[27] A. Filho, D. Garcia, F. Nascimento, J. Angarita, "Study of voltage unbalance conditions based on the behavior of the complex voltage unbalance factor (CVUF)," IEEE Proc. Transmission and Distribution Conference and Exposition, Sao Paulo, Brazil, pp. 184 - 189, 2010.

[28] V. Subba Rao, "Balancing bundle conductor transmission line constants without transpositions," Proc. of the Institution of Electrical Engineers, Vol. 112, No. 5, pp. 931 - 940, 1965.

[29] C-Y. Lee, "Effects of unbalanced voltage on the operation of a three-phase induction motor," IEEE Trans. on Energy Conversion, Vol. 14, No. 2, pp. 202-208, 1999.

[30] R. F. Woll, "Effect of unbalanced voltage on the operation of polyphase induction motors," IEEE Trans. on Industry Applications, Vol. 11, No. 1, pp. 38 - 42, 1975.

[31] K. Olejniczak, G. Heydt, "Basic mechanisms of generation and flow of harmonic signals in balanced and unbalanced three-phase systems," IEEE Trans. on Power Delivery, Vol. 4, No. 4, pp. 2162 $2170,1989$. 